\title{
THE FIRST DETECTION OF NEAR-INFRARED CN BANDS IN ACTIVE GALACTIC NUCLEI: SIGNATURE OF STAR FORMATION
}

\author{
R. RifFel and M. G. PAstoriza \\ Departamento de Astronomia, Universidade Federal do Rio Grande do Sul, Av. Bento Gonçalves 9500, \\ CP 15051, CEP 91501-970 Porto Alegre RS, Brazil; riffel@ufrgs.br \\ A. RodrígueZ-ARDILA ${ }^{1}$ \\ Laboratório Nacional de Astrofísica, Rua dos Estados Unidos 154, Bairro das Nações, CEP 37504-364 Itajubá MG, Brazil \\ AND \\ C. MARASTON \\ University of Oxford, Denys Wilkinson Building, Keble Road, Oxford OX1 3RH, UK \\ Received 2007 February 26; accepted 2007 March 12; published 2007 March 20
}

\begin{abstract}
We present the first detection of the near-infrared $\mathrm{CN}$ absorption band in the nuclear spectra of active galactic nuclei (AGNs). This feature is a recent star formation tracer, being particularly strong in carbon stars. The equivalent width of the $\mathrm{CN}$ line correlates with that of the $\mathrm{CO}$ at $2.3 \mu \mathrm{m}$ as expected in stellar populations (SPs) with ages between $\sim 0.2$ and $\sim 2$ Gyr. The presence of the $1.1 \mu \mathrm{m} \mathrm{CN}$ band in the spectra of the sources is taken as unambiguous evidence of the presence of young/intermediate SPs close to the central source of the AGNs. Near-infrared bands can be powerful age indicators for star formation connected to AGNs, the understanding of which is crucial in the context of galaxy formation and AGN feedback.
\end{abstract}

Subject headings: galaxies: active — galaxies: nuclei — galaxies: stellar content

Online material: color figures

\section{INTRODUCTION}

It is widely known that circumnuclear star formation is commonly detected in active galactic nuclei (AGNs; e.g., Sturm et al. 1999; Contini et al. 2002; Storchi-Bergmann et al. 2005; Shi et al. 2006). In fact, in the last few years, increasing observational evidence has confirmed that nuclear/circumnuclear starbursts (SBs) coexists in objects harboring an AGN (e.g., Mizutani et al. 1994; Imanishi \& Dudley 2000; Imanishi 2002; Rodríguez-Ardila \& Viegas 2003; Imanishi \& Wada 2004). For example, Storchi-Bergmann et al. (2000), in a study of a sample of 20 Seyfert 2 (Sy2) galaxies, find that about $50 \%$ of the sources have young to intermediate-aged ( 1 Gyr) nuclear starbursts and that $30 \%$ of the galaxies display a recent burst of star formation $(t<500 \mathrm{Myr}$; results confirmed by GonzálezDelgado et al. 2001). Currently, it is thought that both the active nucleus and the starburst might be related to gas inflow, probably triggered by an axis-asymmetry perturbation like bars, mergers, or tidal interactions (Shlosman et al. 1989, 1990; Maiolino et al. 1997; Knapen et al. 2000; Fathi et al. 2006). This gives support to the so-called AGN-SB connection (Norman \& Scoville 1988; Terlevich et al. 1990; Heckman et al. 1997; González Delgado et al. 1998; Ferrarese \& Merritt 2000; Veilleux 2001; Heckman 2004). This connection, however, could be incidental, as many Seyfert galaxies do not show any evidence of starburst activity (e.g., Filippenko et al. 1993) and as optical spectroscopic studies of large samples do not indicate that starbursts are more common in Seyfert galaxies than in normal galaxies (Pogge 1989). One difficulty in establishing the AGN-SB connection further is that tracing starbursts reliably is difficult (Oliva et al. 1995). In the NIR region, except for a few indicators such as the methods based on the $\mathrm{CO}(2-$

\footnotetext{
${ }^{1}$ Visiting Astronomer at the Infrared Telescope Facility, which is operated by the University of Hawaii under cooperative agreement NCC 5-538 with the National Aeronautics and Space Administration, Science Mission Directorate, Planetary Astronomy Program.
}

0) first overtone or the $\mathrm{Br} \gamma$ emission (e.g., Origlia et al. 1993; Oliva et al. 1995), the detection of spectral features allowing for the identification and dating of young stellar populations (SPs) in the inner few tens of parsecs of AGNs remains difficult. To understand the physics of the above connection is particularly important for galaxy formation theories, as AGN activity is advocated as the regulator of star formation in massive protogalaxies, the so-called AGN feedback phenomenon (Silk \& Rees 1998; Binney 2004; Croton et al. 2006; Bower et al. 2006).

In this line of thought, the evolutionary population synthesis calculations presented by Maraston (2005, hereafter M05), by including empirical spectra of carbon- and oxygen-rich stars (Lançon \& Wood 2000), are able to foresee the presence of molecular features like $\mathrm{CH}, \mathrm{CN}$, and $\mathrm{C}_{2}$. They arise in young/ intermediate SPs, and their spectral signatures are particularly enhanced in the NIR. Of particular importance are the CN bands, which arise according to the models, from stars with ages in the range $0.3 \mathrm{Gyr} \leq t \leq 2 \mathrm{Gyr}$, and are attributed to stars in the thermally pulsing asymptotic giant branch (TPAGB) phase. This means that their detection can be taken as an unambiguous signature of the presence of young/intermediate SPs in a well-constrained age, signaling the occurrence of SB activity. In the optical, $\mathrm{CN}$ bands are common in the spectra of globular clusters (e.g., Smith et al. 1989; Cohen 1999a, 1999b; Smith \& Briley 2006) but have rarely been reported in AGNs. To our knowledge, the object showing the presence of this feature is the Sy2 galaxy NGC 7679, for which Gu et al. (2001) report an extremely weak optical CN $\lambda 4200$ line. However, contrary to the NIR, optical CN reveals old SPs rather than young/intermediate-age ones (e.g., Storchi-Bergmann et al. 2000). Here we report the first observation of the $1.1 \mu \mathrm{m} \mathrm{CN}$ band predicted by the M05 models in a comprehensive sample of Seyfert galaxies, demonstrating the usefulness of the NIR spectral region as an important tool for investigating recent star formation episodes.

This Letter is structured as follows: In $\S 2$ we describe the 
TABLE 1

Subsample of RRP06 Sample Showing the Presence of the NIR CN MoleCUlar Band

\begin{tabular}{cccccc}
\hline \hline ID & Galaxy & $\begin{array}{c}\text { Type } \\
(1)\end{array}$ & $\begin{array}{c}r^{\mathrm{a}} \\
(\mathrm{pc})\end{array}$ & $\begin{array}{c}W_{\mathrm{CN}} 1.1 \mu \mathrm{m} \\
(\mathrm{mag}) \\
(4)\end{array}$ & $\begin{array}{c}W_{\mathrm{CO}} 2.29 \mu \mathrm{m} \\
(\mathrm{mag}) \\
(6)\end{array}$ \\
\hline $1 \ldots \ldots$. & Mrk 334 & Sy1 & 340 & $0.063 \pm 0.001$ & $0.047 \pm 0.003$ \\
$2 \ldots \ldots$. & NGC 34 & SB/Sy2 & 230 & $0.082 \pm 0.001$ & $0.117 \pm 0.001$ \\
$3 \ldots \ldots$. & NGC 591 & Sy2 & 206 & $0.055 \pm 0.001$ & $0.098 \pm 0.009$ \\
$4 \ldots \ldots$. & Mrk 573 & Sy2 & 267 & $0.046 \pm 0.002$ & $0.057 \pm 0.005$ \\
$5 \ldots \ldots$. & NGC 1097 & Sy1 & 58 & $0.019 \pm 0.002$ & $0.078 \pm 0.005$ \\
$6 \ldots \ldots$. & NGC 1144 & Sy2 & 447 & $0.062 \pm 0.002$ & $0.077 \pm 0.009$ \\
$7 \ldots \ldots$. & Mrk 1066 & Sy2 & 186 & $0.045 \pm 0.001$ & $0.097 \pm 0.007$ \\
$8 \ldots \ldots$. & NGC 1614 & SB & 154 & $0.085 \pm 0.002$ & $0.139 \pm 0.007$ \\
$9 \ldots \ldots$. & NGC 2110 & Sy2 & 121 & $0.066 \pm 0.001$ & $0.033 \pm 0.001$ \\
$10 \ldots \ldots$ & NGC 3310 & SB & 56 & $0.052 \pm 0.002$ & $0.080 \pm 0.009$ \\
$11 \ldots \ldots$. & NGC 5929 & Sy2 & 193 & $0.050 \pm 0.002$ & $0.082 \pm 0.009$ \\
$12 \ldots \ldots$. & NGC 5953 & Sy2 & 165 & $0.041 \pm 0.002$ & $0.104 \pm 0.002$ \\
$13 \ldots \ldots$. & NGC 7682 & Sy2 & 179 & $0.036 \pm 0.001$ & $0.079 \pm 0.009$ \\
$14 \ldots \ldots$ & NGC 7714 & H II & 115 & $0.081 \pm 0.002$ & $0.098 \pm 0.021$ \\
\hline
\end{tabular}

Note. - Some of the galactic properties and the equivalent widths of the $\mathrm{CN}$ band and the $\mathrm{CO}$ line are also presented.

${ }^{\text {a }}$ Radius of the integrated region. data. The results are presented in $\S 3$. A discussion of the results is made in $\S 4$. Final remarks are given in $\S 5$.

\section{THE DATA}

The galaxy spectra chosen for this work are the ones presented by Riffel et al. (2006, hereafter RRP06). The NIR spectra were obtained at the NASA Infrared Telescope Facility using the $3 \mathrm{~m}$ infrared optimized telescope. SpeX (a medium-resolution 0.8$5.5 \mu \mathrm{m}$ cryogenic spectrograph and imager; Rayner et al. 2003) was used in the short cross-dispersed mode. A $0.8^{\prime \prime} \times 15^{\prime \prime}$ slit was employed giving a spectral resolution of $360 \mathrm{~km} \mathrm{~s}^{-1}$. For more details see RRP06. A rapid inspection of the left panels of Figures 1-8 and Figure 12 of RRP06 shows that a significant fraction of objects display a deep prominent broad absorption feature starting at $\sim 1.1 \mu \mathrm{m}$ and extending up to $1.15 \mu \mathrm{m}$ in some sources (see, e.g., NGC 1097, NGC 1144, and NGC 1614). In other objects such as Mrk 1066, NGC 5953, and NGC 7714, it is considerably narrower but still deep and strong. Almost invariably, the galaxies displaying the $1.1 \mu \mathrm{m}$ absorption also present strong $\mathrm{CO} 2.3 \mu \mathrm{m}$ bands and a $H$-continuum dominated by several absorption lines. The CaT at $0.8 \mu \mathrm{m}$ is also prominent in these objects. Moreover, these sources were classified as Sy2/ SB, except for Mrk 334 and NGC 1097, which were considered
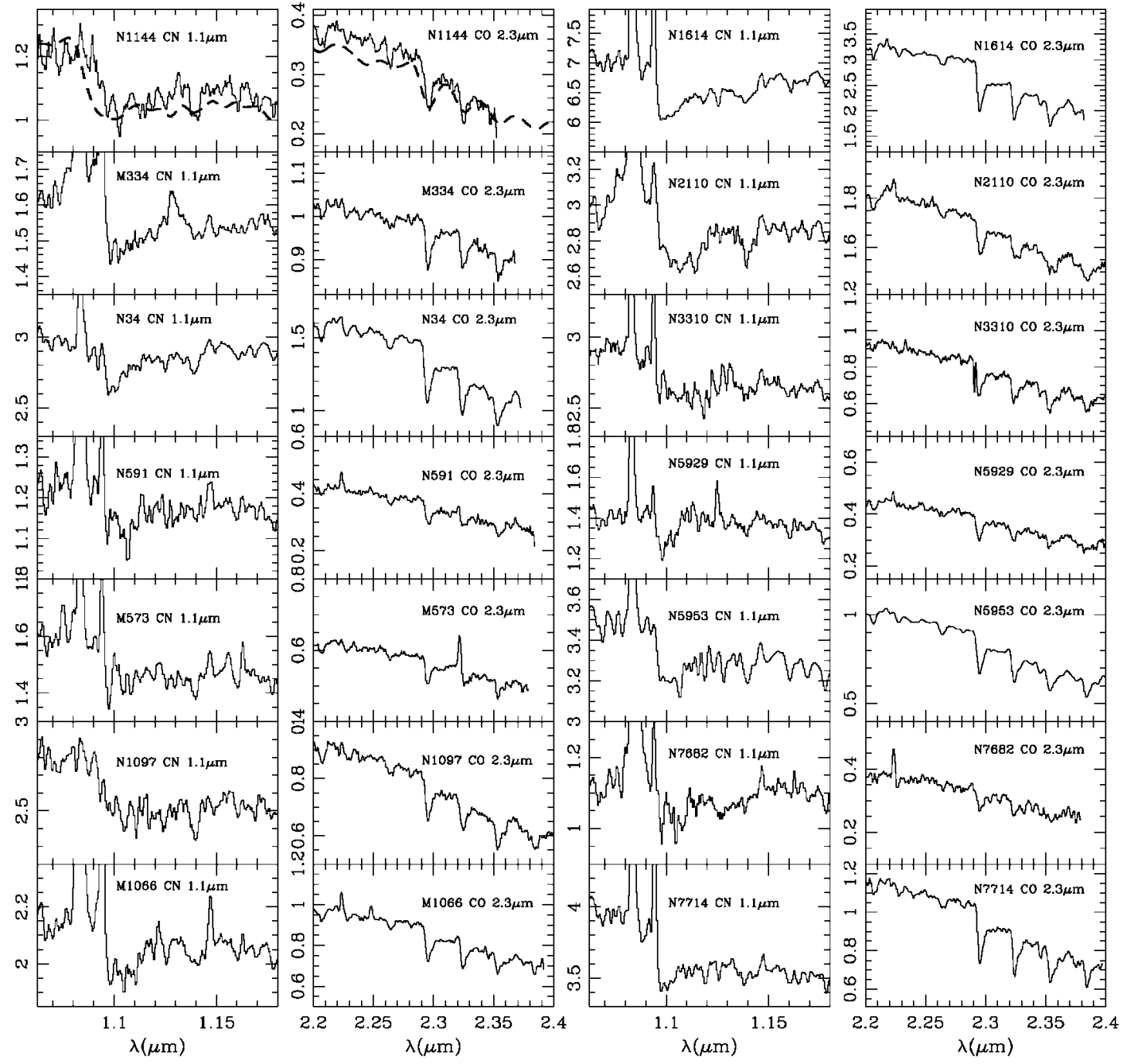

FIG. 1.-Zoom of the $1.1 \mu \mathrm{m} \mathrm{CN}$ band and the $2.2 \mu \mathrm{m} \mathrm{CO}$ band for galaxies that clearly show the presence of the $1.1 \mu \mathrm{m}$ CN band. In the top left panel, we show the predicted $1.1 \mu \mathrm{m} \mathrm{CN}$ band of a population with a $1 \mathrm{Gyr}$ and solar metallicity template (M05) overplotted on the NGC 1144 spectrum and the CN molecular band lines observed in our sample of galaxies. The ordinate is the flux in units of $10^{-15} \mathrm{ergs} \mathrm{cm}^{-2} \mathrm{~s}^{-1}$. 


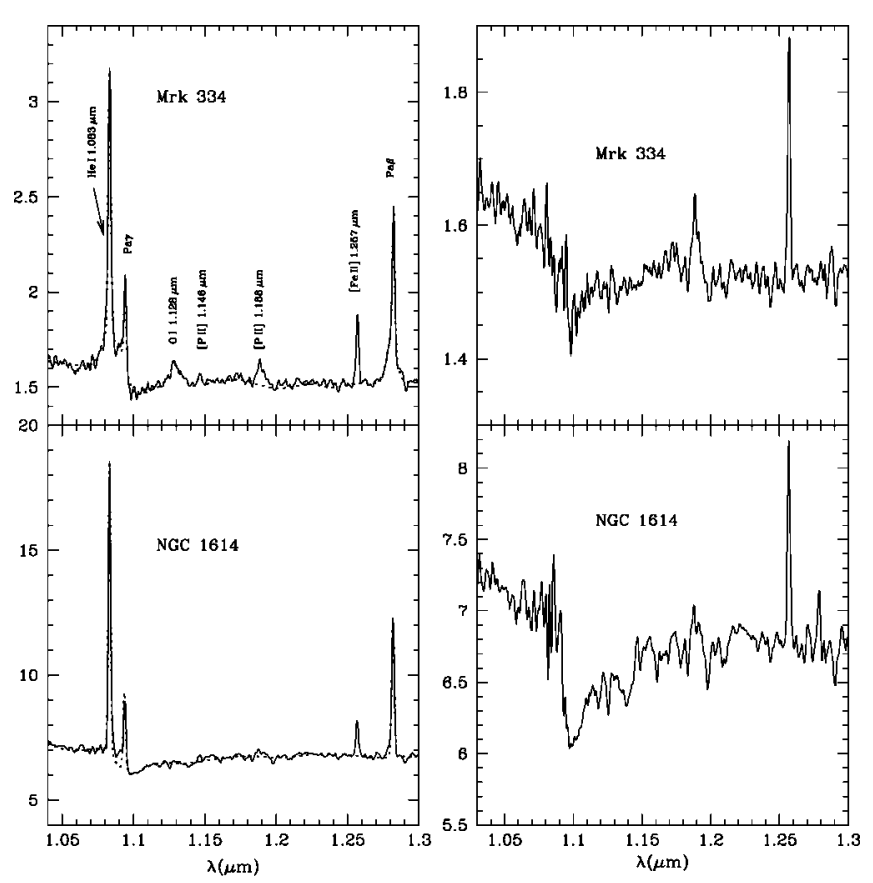

FIG. 2.-Model for the emission lines around the $\mathrm{CN}$ absorption band. In the left panels, the solid lines are the spectra of the galaxies, and the modeled lines and continua are represented as dotted lines. In the right panels, the $\mathrm{CN}$ band, free from the emission spectra, is presented. The ordinate is the flux in units of $10^{-15} \mathrm{ergs} \mathrm{cm}^{-2} \mathrm{~s}^{-1}$. [See the electronic edition of the Journal for a color version of this figure.]

Sy1 galaxies by RRP06. The invariable association with $\mathrm{CO}$ bands and the CaT triplet allowed us to consider that the $1.1 \mu \mathrm{m}$ absorption could indeed be associated with the stellar population, although no previous observational report of this feature was found in the literature.

Columns (2) and (3) of Table 1 list the names and AGN type, respectively, of the galaxies where clear evidence of $1.1 \mu \mathrm{m}$ absorption was found.

\section{RESULTS}

The association of the $1.1 \mu \mathrm{m}$ absorption with the $\mathrm{CN}$ molecule was evident after comparing our spectra with Figure 15 of M05, where a grid of synthetic spectra that include the effects of TP-AGB stars is shown. In these models, the dominant feature in the interval $0.9-1.4 \mu \mathrm{m}$ is a strong absorption centered at $1.1 \mu \mathrm{m}$ and attributed to $\mathrm{CN}$. Models that do not include the TP-AGB population do not display the absorption. The M05 models also show that the strength of the $\mathrm{CN}$ absorption is a function of age and metallicity (Fig. 15 of M05). Other features present in the templates are also easily recognized in our data, including the overall shape of the continuum emission and the CaT and CO bands, particularly in the SB galaxies.

The top left panel of Figure 1 shows a comparison of a 1 Gyr and solar metallicity template of M05 with our data, confirming that the $1.1 \mu \mathrm{m}$ absorption is due to $\mathrm{CN}$, associated with TP-AGB stars in the galaxies. No previous report of the CN $1.1 \mu \mathrm{m}$ band in extragalactic objects was made before. Our result points out the presence of a significant young/intermediate stellar population, with ages between 0.3 and 2 Gyr in the objects listed in Table 1.

In order to properly quantify the strength of the $\mathrm{CN} 1.1 \mu \mathrm{m}$ band, we need to subtract the emission lines of He I $\lambda 1.0830 \mu \mathrm{m}$ and $\mathrm{Pa} \gamma 1.0938 \mu \mathrm{m}$ that affect the band. Therefore, we have modeled these emission lines by constraining the Par width and

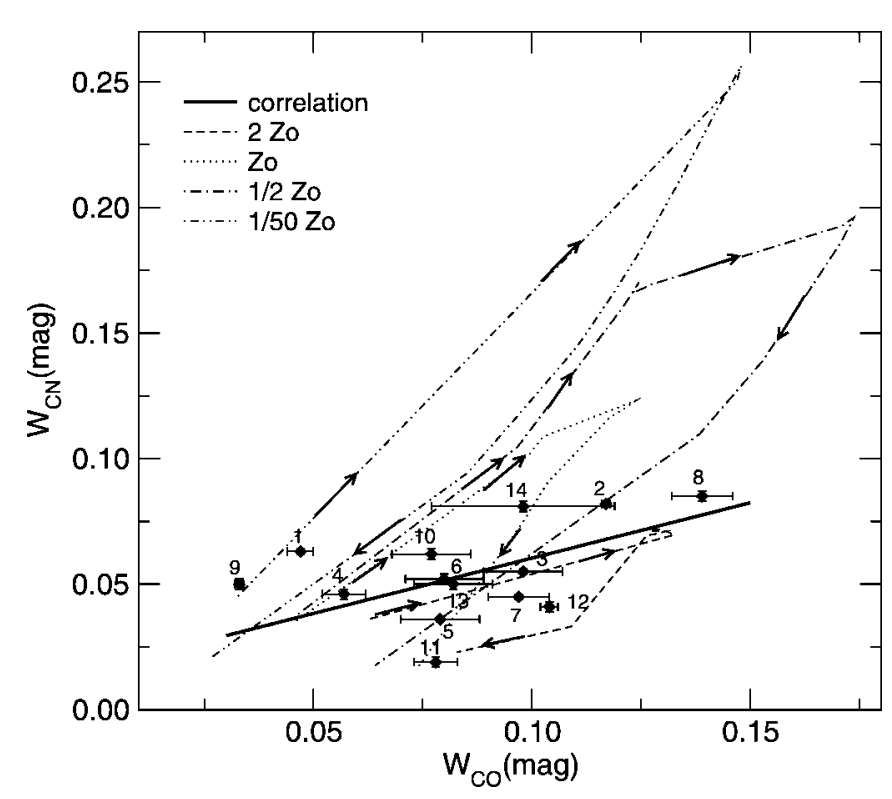

FIG. 3. - CN equivalent width vs. CO equivalent width. The observed values are represented as filled circles. The numbers identify the sources according to col. (1) of Table 1 . The models are indicated. The arrows indicate the age sequence of the models ranging from 0.2 to $3 \mathrm{Gyr}$. [See the electronic edition of the Journal for a color version of this figure.]

flux to those of $\mathrm{Pa} \beta 1.2820 \mu \mathrm{m}$ using the calculations of Hummer $\&$ Storey (1987) for their intrinsic line ratio values. For He I, we have adjusted a Gaussian to the emission-line profile. It is a reliable approximation because the helium emission line is located near the blue end of the $\mathrm{CN}$ absorption band. As for He $\mathrm{I}$, in the spectrum of Mrk 334 we have adjusted a Gaussian to the O I $\lambda 1.1287 \mu \mathrm{m}$ emission line. The LINER software (Pogge \& Owen 1993) was used for this purpose. A sample of the results of this process is presented in Figure 2.

With a clear $\mathrm{CN}$ band, free of contamination of the emission lines, we proceed to measure the equivalent width $(W)$ of the $\mathrm{CN}$ band. The continuum was linearly adjusted using the points between 1.0445 and $1.0580 \mu \mathrm{m}$ as representative of the continuum at the left side of the $\mathrm{CN}$ band, and for the right side we defined the points between 1.2160 and $1.2385 \mu \mathrm{m}$. The bandwidths were defined using the Maraston models, as being between 1.0780 and $1.1120 \mu \mathrm{m}$. We also include, for comparison, the $W$ of the CO $2.2980 \mu \mathrm{m}$ line of the $2.3 \mu \mathrm{m} \mathrm{CO}$ band, measured from the galaxy spectra, taking as the line width the points 2.2860 and $2.3100 \mu \mathrm{m}$ for the bandwidth and a continuum defined as a spline using points free from emission/ absorption lines in the interval between 2.2350 and $2.3690 \mu \mathrm{m}$. The $W_{\mathrm{CN}}$ and $W_{\mathrm{CO}}$ measured values are listed in columns (7) and (8) of Table 1. Here the LINER software was also used.

\section{DISCUSSION}

The NIR CN absorption band is particularly enhanced in carbon stars, where there is residual carbon that is not bound with oxygen in $\mathrm{CO}$ molecules. As carbon stars are only produced after the third convective dredge-up along the TP-AGB phase (e.g., Iben \& Renzini 1983), their features are a clear indicator of SPs rich in these types of stars. This constrains the age of such SPs to be within the narrow range of 0.3-2 Gyr (M05).

In order to check whether or not our observations are actually consistent with this claim, we compare the observed strengths of $\mathrm{CN}$ and $\mathrm{CO}$ with Maraston's model predictions. Note that $\mathrm{CO}$ alone would not be a useful age indicator because this band 
is not only strong in TP-AGB stars and red supergiants, but also in old RGB stars (e.g., Ivanov et al. 2000; Imanishi \& Alonso-Herrero 2004). In Figure 3, we plot the values of simple stellar populations with ages between 0.2 and 3 Gyr and metallicities from $1 / 50$ to twice solar.

Figure 3 confirms that the observed values are indeed consistent with the model predictions in this age range and that the strengths of the two bands are correlated $\left(R^{2}=0.66\right)$. The $\mathrm{CO}$ band is also present in TP-AGB spectra (see Fig. 16 of M05), which explains the correlation between the $W$ of $\mathrm{CN}$ and $\mathrm{CO}$. Interestingly, the data can be separated into two main groups according to chemical composition. One group (NGC 34, Mrk 573, NGC 1144, NGC 3310, NGC 7682, and NGC 7714) has light dominated by stars with solar/half-solar metallicity. A second group (NGC 591, NGC 1097, Mrk 1066, NGC 5929, and NGC 5953) harbors SPs with high metallicity, and just two objects are consistent with low metallicity. A detailed analysis of the ages and metallicities of our sample is postponed to another paper (R. Riffel et al. 2007, in preparation).

Optical stellar population synthesis carried out by Raimann et al. (2003) in a sample of Seyfert galaxies, four of them common to our sample (Mrk 573, Mrk 1066, NGC 2110, and NGC 5929), reveals that an old population (10 Gyr) dominates the inner few hundred parsecs. The integrated regions of our NIR spectra are similar to that of Raimann et al. (2003). As shown by our results, for these same objects the NIR spectra display an unambiguous signature of young/intermediate SPs with ages between 0.3 and 2 Gyr. This means that the $J$ band provides complementary information not detected in the op- tical. An additional advantage of the $\mathrm{CN}$ is that it is located near the center of the $J$ band, allowing for its observation in a wide range of redshifts $(z \lesssim 0.18)$. The $\mathrm{CO}$ band is instead located near the red end of the $K$ band, thereby allowing only observations of sources with redshifts smaller than 0.03 .

\section{FINAL REMARKS}

We present the first detection of the $1.1 \mu \mathrm{m} \mathrm{CN}$ band in the inner $300 \mathrm{pc}$ of an AGN. The presence of the $\mathrm{CN}$ at $1.1 \mu \mathrm{m}$ in the spectrum can be taken as unambiguous evidence of recent star formation; in particular, it is associated with the presence of bright carbon stars. We have compared the observed values with predictions of SPs including the contribution of carbon stars. We find a nice consistency of observed and predicted values for ages around $1 \mathrm{Gyr}$. This supports the claim that the NIR CN band is suggestive of young/intermediate-age SPs. Its use as an age indicator is particularly adaptable to AGN hosts, where dust reddening can complicate seriously the use of optical bands. Moreover, since these molecules are enhanced in SPs spanning a narrow age range, their detection is relatively robust against the age/metallicity degeneracy.

This Letter was partially supported by the Brazilian funding agency CNPq (473465/2004-3 and 311476/2006-6) to A. Rodríquez-Ardila. C. Maraston is a Marie Curie Excellence Team Leader and holds grant MEXT-CT-2006-1 042754 of the Training and Mobility of Researchers programme financed by the European Community.

\section{REFERENCES}

Binney, J. 2004, MNRAS, 347, 1093

Bower, R. G., Benson, A. J., Malbon, R., Helly, J. C., Frenk, C. S., Baugh, C. M., Cole, S., \& Lacey, C. G. 2006, MNRAS, 370, 645

Cohen, J. G. 1999a, AJ, 117, 2428

$$
\text { 1999b, AJ, 117, } 2434
$$

Contini, M., Radovich, M., Rafanelli, P., \& Richter, G. M. 2002, ApJ, 572, 124

Croton, D. J., et al. 2006, MNRAS, 365, 11

Fathi, K., Storchi-Bergmann, T., Riffel, R. A., Winge, C., Axon, D. J., Robinson, A., Capetti, A., \& Marconi, A. 2006, ApJ, 641, L25

Ferrarese, L., \& Merritt, D. 2000, ApJ, 539, L9

Filippenko, A. V., Ho, L. C., \& Sargent, W. L. W. 1993, ApJ, 410, L75

González Delgado, R. M., Heckman, T., \& Leitherer, C. 2001, ApJ, 546, 845

González Delgado, R. M., Heckman, T., Leitherer, C., Meurer, G., Krolik, J.,

Wilson, A. S., Kinney, A., \& Koratkar, A. 1998, ApJ, 505, 174

Gu, Q. S., Huang, J. H., de Diego, J. A., Dultzin-Hacyan, D., Lei, S. J., \& Benitez, E. 2001, A\&A, 374, 932

Heckman, T. M. 2004, in Coevolution of Black Holes and Galaxies, ed. L. C. Ho (Cambridge: Cambridge Univ. Press), 358

Heckman, T. M., Gonzalez-Delgado, R. M., Leitherer, C., Meurer, G. R., Krolik, J., Wilson, A. S., Koratkar, A., \& Kinney, A. 1997, ApJ, 482, 114

Hummer, D. G., \& Storey, P. J. 1987, MNRAS, 224, 801

Iben, I., Jr., \& Renzini, A. 1983, ARA\&A, 21, 271

Imanishi, M. 2002, ApJ, 569, 44

Imanishi, M., \& Alonso-Herrero, A. 2004, ApJ, 614, 122

Imanishi, M., \& Dudley, C. C. 2000, ApJ, 545, 701

Imanishi, M., \& Wada, K. 2004, ApJ, 617, 214

Ivanov, V. D., Rieke, G. H., Groppi, C. E., Alonso-Herrero, A., Rieke, M. J., \& Engelbracht, C. W. 2000, ApJ, 545, 190

Knapen, J. H., Shlosman, I., \& Peletier, R. F. 2000, ApJ, 529, 93
Lançon, A., \& Wood, P. R. 2000, A\&AS, 146, 217

Maiolino, R., Ruiz, M., Rieke, G. H., \& Papadopoulos, P. 1997, ApJ, 485, 552 Maraston, C. 2005, MNRAS, 362, 799 (M05)

Mizutani, K., Suto, H., \& Maihara, T. 1994, ApJ, 421, 475

Norman, C., \& Scoville, N. 1988, ApJ, 332, 124

Oliva, E., Origlia, L., Kotilainen, J. K., \& Moorwood, A. F. M. 1995, A\&A, 301,55

Origlia, L., Moorwood, A. F. M., \& Oliva, E. 1993, A\&A, 280, 536

Pogge, R. W. 1989, ApJS, 71, 433

Pogge, R. W., \& Owen, J. M. 1993, OSU Internal Rep. 93-01

Raimann, D., Storchi-Bergmann, T., González Delgado, R. M., Cid Fernandes, R., Heckman, T., Leitherer, C., \& Schmitt, H. 2003, MNRAS, 339, 772

Rayner, J. T., Toomey, D. W., Onaka, P. M., Denault, A. J., Stahlberger, W. E., Vacca, W. D., Cushing, M. C., \& Wang, S. 2003, PASP, 115, 362

Riffel, R., Rodríguez-Ardila, A., \& Pastoriza, M. G. 2006, A\&A, 457, 61 (RRP06)

Rodríguez-Ardila, A., \& Viegas, S. M. 2003, MNRAS, 340, L33

Shi, L., Gu, Q. S., \& Peng, Z. X. 2006, A\&A, 450, 15

Shlosman, I., Begelman, M. C., \& Frank, J. 1990, Nature, 345, 679

Shlosman, I., Frank, J., \& Begelman, M. C. 1989, Nature, 338, 45

Silk, J., \& Rees, M. J. 1998, A\&A, 331, L1

Smith, G. H., Bell, R. A., \& Hesser, J. E. 1989, PASP, 101, 1083

Smith, G. H., \& Briley, M. M. 2006, PASP, 118, 740

Storchi-Bergmann, T., Nemmen, R. S., Spinelli, P. F., Eracleous, M., Wilson, A. S., Filippenko, A. V., \& Livio, M. 2005, ApJ, 624, L13

Storchi-Bergmann, T., Raimann, D., Bica, E. L. D., \& Fraquelli, H. A. 2000, ApJ, 544, 747

Sturm, E., et al. 1999, ApJ, 512, 197

Terlevich, E., Diaz, A. I., \& Terlevich, R. 1990, MNRAS, 242, 271

Veilleux, S. 2001, in Starburst Galaxies: Near and Far, ed. L. Tacconi \& D. Lutz (Heidelberg: Springer), 88 